\title{
Impact of the Primary Network Activity on the Maximum Achievable Capacity of DS-CDMA/OFDM Spectrum Sharing
}

\author{
Mohammad G. Khoshkholgh, Keivan Navaie \\ Department of Electrical and Computer Engineering \\ Tarbiat Modares University, Tehran, Iran \\ Email: keivan.navaie@ieee.org
}

\author{
Halim Yanikomeroglu \\ Systems and Computer Engineering Department \\ Carleton University, Ottawa, Canada \\ Email: halim@sce.carleton.ca
}

\begin{abstract}
In this paper, we analyze DS-CDMA/OFDM spectrum sharing system based on opportunistic spectrum access (OSA). In this system, the primary network air interface is based on DS-CDMA, in which the system performance is limited by a maximum total received interference. The total interference, among other things, is a function of the primary users' communication activity. In OSA, part of this interference is considered to be created by the secondary service, this portion is called interference threshold. Decreasing the activity of the primary users results in a higher interference threshold, and at the same time, creates less interference at the secondary service receiver; thus the achievable capacity of the secondary service is increased. In this paper, we analytically obtain the maximum achievable capacity of the secondary service over fading channel. Our analysis reveals the dependency of the achievable capacity and the users' activity in the primary network. A direct consequence of our analysis is that exploiting the temporal variations of the available radio resource caused by the users' activity in the primary network can significantly increase the maximum achievable capacity. Simulation results confirm our analysis.
\end{abstract}

\section{INTRODUCTION}

Spectrum sharing was first proposed by the Federal Communications Commission (FCC) [1] to improve the utilization of the allocated frequency bands. In this method, under certain conditions, a Secondary Service is able to access to a frequency band formally allocated to the Primary Service [2]. Various schemes are proposed in the literature for spectrum sharing (see e.g., [3] and references therein). In this paper, our focus is the Opportunistic Spectrum Access (OSA). In OSA the secondary service dynamically detects and makes use of the spectrum holes [4]. Spectrum holes are those parts of the spectrum allocated to the primary user which are under-utilized in some particular times and specific locations. Clearly, spectrum sharing must not cause Quality-of-Service degradation to the primary service.

In this paper, as a metric for recognizing a spectrum hole we consider a threshold on the acceptable level of the imposed interference caused by the operation of the secondary user. Therefore, a spectrum hole is defined as a frequency band in which the interference level is below the Interference Threshold.

In the spectrum sharing, the under-utilized spectrum as the available radio resource which can be utilized by the secondary service varies over time. Given an acceptable level of accuracy in the spectrum sensing, the temporal variations can be then exploited subject to the primary system interference threshold. Therefore, the maximum achievable capacity is directly affected by the temporal variations in the available radio resources. It is shown in [5] and [6] that obtaining the maximum achievable capacity for an Additive White Gaussian Noise (AWGN) channel in different transmission scenarios with interference threshold is an instant of the water filling problem.

Different parameters have impacts on the temporal variation of the available radio resource in a spectrum sharing system including the dynamic behavior of wireless channels, as well as the activity of the users in the primary network. User activity in the primary network includes two important parameters, the number of active users, and their corresponding traffic profile. Under various fading conditions, the maximum achievable capacity is studied in [7]. It is shown in [7] that employing OSA results in a significant increase in the achievable capacity in comparison with the OSA in an equivalent AWGN channel.

In most of the previous works the primary users' activity is simply ignored by assuming constant bit-rate transmission. However, in practise most of the data services are bursty in nature; therefore, the constant activity assumption is questionable. In other words, activity of the users in the primary networks have impact on the fraction of time (or the level of transmission power) in which the secondary service is able to access to the spectrum, thus on the maximum achievable capacity. In this paper, our main objective is to analyze the impact of the users' activity in the primary network on the maximum achievable capacity.

In this paper, we focus on the DS-CDMA/OFDM spectrum sharing, in which the primary network air interface is based on DS-CDMA and the secondary service utilizes OFDM for spectrum sharing. DS-CDMA is the dominant air interface technique for the third generation (3G) mobile communications and some Wireless Local Area Network (WLAN) technologies. Therefore, the spectrum sharing over existing DS-CDMA-based networks is anticipated to be one of important spectrum sharing applications in the near future. The secondary service employs OSA. Utilizing OFDM by the secondary service provides the required flexibility for the secondary service in terms of access over appropriate portions of the spectrum. In our model, the spectrum is divided into a number of sub-channels and then the secondary service makes 
access to one (or more) of these sub-channels, subject to the interference threshold constraint. Note that the secondary service is able to access the spectrum currently in use while primary service is also active subject to complying with the interference threshold.

We analytically obtain the maximum achievable capacity of the secondary service over fading channel. Our analysis reveals the dependency of the achievable capacity and the users' activity in the primary network. Decreasing the activity of the primary users results in a higher interference threshold, and at the same time, creates less interference at the secondary service receiver; thus the achievable capacity of the secondary service is increased. A direct consequence of the results presented in this paper is that adopting a method by the secondary service to efficiently exploit the temporal variations of the available radio resource (i.e., acceptable interference), can significantly increase the maximum achievable capacity of OSA systems. The temporal variations are mainly due to the traffic characteristics of the primary users as well as their corresponding wireless channel temporal dynamics.

The rest of this paper is organized as follows. In Section II, the system model is presented. In Section III, we model users' activity in the primary network. Then we obtain the maximum achievable capacity in Section IV. Simulation results are presented in Section V, followed by some practical considerations and conclusions in Section VI.

\section{SySTEM MODEL}

In spectrum sharing, two services try to access a $B \mathrm{~Hz}$ spectrum band: Primary Service and Secondary Service. The frequency band has been licensed to the primary service. The primary service is provided by the Primary Network to the Primary Users. The secondary service does not have the spectrum license, but may acquire access to the spectrum by adopting OSA. The secondary service utilizes OFDM to access the spectrum.

Since in this paper we focus on the impact of the primary service activity on the maximum achievable capacity of the secondary service, for brevity of expositions we consider one secondary service user which we simply refer to as secondary user. Of course in cases where number of secondary users is more than one, depending on the access strategy, the inter-operation among them may reduce the capacity and the obtained capacity bound remains valid.

The wireless channel is a point-to-point frequency-selective AWGN channel with coherence bandwidth of $B_{c} \mathrm{~Hz}$. The white noise power spectral density is $N_{0}$. The channel is divided into $N$ flat fading $B_{c} \mathrm{~Hz}$ sub-channels. Sub-channels are indexed by $i=1,2, \ldots, N$. Based on the primary network status, the secondary service may have access to $M$ accessible sub-channels indexed by $j=0,1, \ldots, M$ where $0 \leq M \leq N$. Based on the adopted OSA scenario, the number of accessible sub-channels can be either adaptively estimated based on the network status or pre-assigned by the primary network or regulator.

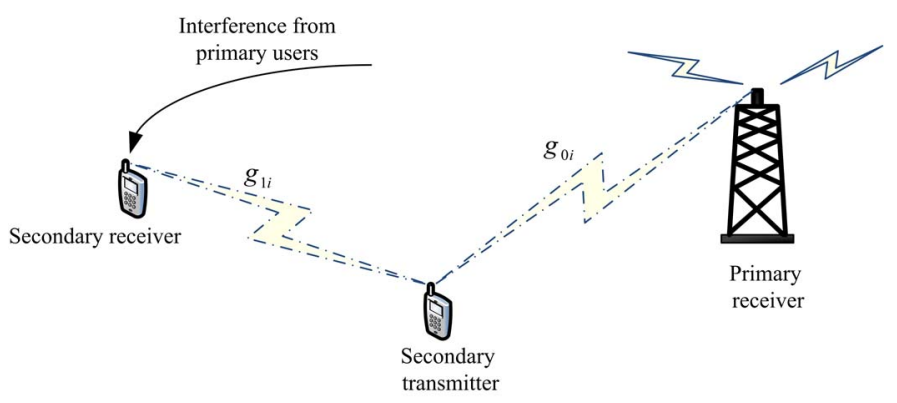

Fig. 1. Spectrum sharing structure for sub-channel $i$

The sub-channel gains are independent and identically distributed (i.i.d.) random processes. Subscripts $s$ and $p$ are used in this paper for the secondary service and the primary service, respectively. Hereafter, we simply refer to "primary spectrum" as "spectrum" unless otherwise stated.

Operation of the secondary service in the spectrum implies interference on the primary service users. The interference threshold, $Q$, is the maximum allowable average interference in the receiver of the primary service caused by the operation of the secondary service in the spectrum.

Fig. 1 shows the schematic diagram of the system considered in this paper. For sub-channel $i, g_{0 i}$ and $g_{1 i}$ in Fig. 1 denote the instantaneous power gains of sub-channel $i$ from the secondary transmitter to the primary and the secondary receivers, respectively. Both $g_{0 i}$ and $g_{1 i}$ are assumed to be stationary and ergodic independent random variables with unit-mean probability density functions (pdf) ${ }^{1}, f_{0 i}\left(g_{0 i}\right)$, and, $f_{1 i}\left(g_{1 i}\right)$, respectively which are i.i.d. for $i=1,2, \ldots, N$.

Since in this paper the main objective is to analyze the impact of the primary users' activity on the maximum achievable capacity, we assume the ideal case where a spectrum coordinator which orchestrates spectrum access exists. The spectrum coordinator thus enables transmitters to have perfect Channel Side Information (CSI) pair $\left(g_{0 i}, g_{1 i}\right), i=1, \ldots, N$. This assumption is usually difficult to implement in practical systems. Thus in practice the achievable capacity is upperbounded by what we obtain in this paper.

The instantaneous transmit power of the secondary transmitter in the $i$ th sub-channel is $P_{s i}\left(g_{0 i}, g_{1 i}\right)$ which we refer to as $P_{s i}$ for brevity. The maximum average transmit power of the secondary transmitter is $\bar{P}_{s}$. To obtain the maximum achievable capacity we also assume that upon accessibility of the spectrum there is always enough backlogged traffic in the secondary service which is ready for transmission.

\section{USERS' ACTIVITY IN THE PRIMARY NETWORK}

As it was mentioned above the available radio resource for spectrum sharing is a function of users' traffic in the primary network characteristics. The term traffic characteristics in a wireless network is usually refereed to the statistics of the number of active users as well as their activity during a call

\footnotetext{
${ }^{1}$ The assumption of unit mean is for brevity of discussions.
} 
or session. For clarity, in this paper we assume that the number of active users in the primary network is constant. Given this, in this paper the traffic characteristics of the primary users is modelled by user's activity. Thus, the term 'activity' refers to the fraction of time a primary user transmits. Note that, in cases where a varying number of active users are in the network coverage area, this model is still able to express the system using a variable activity factor for a fixed number of users.

In DS-CDMA/OFDM, the OFDM secondary service can always access to a part of spectrum as far as the interference threshold constraint is not violated in the receiver of the DSCDMA primary service.

In multi-user DS-CDMA networks the total received interference caused by other active users in the network coverage area is simply modelled as an additive white Gaussian noise with a power spectral density equal to the product of the background noise power spectral density and a factor $(K-1)$ [8]. Since the capacity of DS-CDMA network is interference limited, in these networks for each transmitter, a silence period detector is usually used which detects the silence periods and stops transmitting during these periods. The fraction of these silence period is a function of the users' activity. This technique reduces the interference and results in a capacity gain. To model the user's activity in DS-CDMA network users' activity factor, $\nu$ is defined which is the fraction of time in which the user is transmitting.

Since the secondary user is OFDM, the interference received from the primary service in each $B_{c} \mathrm{~Hz}$ sub-channel can be modelled as an additive white Gaussian noise with power spectral density of $(K-1) N_{0} B_{c}, K \geq 1$. The more activity in the primary network, the higher is the value of $K$.

Using the results presented in our previous work [9] $K$ can be obtained for the uplink of a DS-CDMA network with chip rate $W$, providing $S$ services each with activity factor $\nu_{s}$, required bit-energy to interference plus noise spectral density, $\rho_{s}$, and bit-rate $R_{s}$ as follows:

$$
K=\left(1-(1+f) \sum_{s=1}^{S} n_{s}\left(1+\frac{W}{\rho_{s} \nu_{s} R_{s}}\right)^{-1}\right)^{-1} .
$$

In (1), $f$ is a system parameter which is mainly a function of users' spatial distribution in the network coverage area. As it is seen, for a given number of users of each service type (i.e., given $\left.n_{s}, s=1, \ldots, S\right), K$ in (1) is an increasing function of the users' activity. It is also observed in (1) that for a given set of services (i.e., given $\nu_{s}, s=1, \ldots, S$ ), $K$ is an increasing function of the number of users in the network coverage area. For brevity we assume only one service in the primary network and consider its corresponding activity factor, namely $\nu$, as the primary network activity factor.

\section{MaXimum AChiEVABle CAPACITY}

The secondary service can always make access to a part of spectrum as far as interference threshold constraint is not violated in the receiver of the primary service. Assume that there are $M$ accessible sub-channels for the secondary service. The value of $M$ can be assigned by the regulator or the primary service operator. The maximum achievable capacity of the secondary service over $M$ accessible sub-channels is the solution of the following optimization problem:

$$
\begin{aligned}
C_{s \mid M}=\max _{\mathbf{P}_{s}} \sum_{j=1}^{M} B_{c} \int_{g_{1 j}, g_{0 j}} \log \left(1+\frac{g_{1 j} P_{s j}}{K N_{0} B_{c}}\right) \times \\
f_{1 j}\left(g_{1 j}\right) f_{0 j}\left(g_{0 j}\right) d g_{0 j} d g_{1 j}, \\
\text { s.t. } \sum_{j=1}^{M} \int_{g_{0 j}, g_{1 j}} P_{s j} f_{1 j}\left(g_{1 j}\right) f_{0 j}\left(g_{0 j}\right) d g_{0 j} d g_{1 j} \leq \bar{P}_{s}, \\
\sum_{j=1}^{M} \int_{g_{0 j}, g_{1 j}} g_{0 j} P_{s j} f_{1 j}\left(g_{1 j}\right) f_{0 j}\left(g_{0 j}\right) d g_{0 j} d g_{1 j} \leq G Q .
\end{aligned}
$$

In the above $\mathbf{P}_{s}=\left(P_{s 1}\left(g_{01}, g_{11}\right), \ldots, P_{s M}\left(g_{0 M}, g_{1 M}\right)\right)$, (3) is the average transmission power constraint for the secondary service, $G$ is the processing gain of DS-CDMA primary network and $Q$ is the interference threshold. Constraint (4) implies the primary service's interference threshold in which secondary service's operation in each sub-channel acts as a narrow-band interference after being multiplied by $1 / G$.

In practice, the optimization problem in (2) is solved in the secondary service transmitter based on the CSI pairs $\left(g_{0 j}, g_{1 j}, j=1, \ldots, M\right)$ as well as $Q$. The time-scale of solving this problem should be lower than the coherence time of $g_{0 j}$ and $g_{1 j}$. The value of $Q$ is also varying over time however, the time-scale of its variations is higher than that of channel gains.

The optimization problem in (2) can also be considered as a modified version of the classic Water Filling problem. The optimal power allocation $\mathbf{P}_{s}^{*}$ is then obtained using Lagrange Multipliers method:

$$
\begin{aligned}
& L\left(\mathbf{P}_{s}, \lambda_{1}, \lambda_{2}\right) \\
& =\sum_{j=1}^{M} \int_{g_{1 j}, g_{0 j}} \log \left(1+\frac{g_{1 j} P_{s j}}{K N_{0} B_{c}}\right) f_{1 j}\left(g_{1 j}\right) f_{0 j}\left(g_{0 j}\right) d g_{0 j} d g_{1 j} \\
& -\lambda_{1}\left(\sum_{j=1}^{M} \int_{g_{0 j}, g_{1 j}} P_{s j} f_{1 j}\left(g_{1 j}\right) f_{0 j}\left(g_{0 j}\right) d g_{0 j} d g_{1 j}-\bar{P}_{s}\right) \\
& -\lambda_{2}\left(\sum_{j=1}^{M} \int_{g_{0 j}, g_{1 j}} g_{0 j} P_{s j} f_{1 j}\left(g_{1 j}\right) f_{0 j}\left(g_{0 j}\right) d g_{0 j} d g_{1 j}-G Q\right) .
\end{aligned}
$$

Taking derivation of (5) with respect to $\mathbf{P}_{s}$ and letting the derivation equal to zero, we have

$$
\frac{g_{1 j}}{K N_{0} B_{c}+g_{1 j} P_{s j}^{*}}=\lambda_{1}^{*}+\lambda_{2}^{*} g_{0 j}, \quad j=1,2, \ldots, M .
$$


The optimal power allocation, $\mathbf{P}_{s}^{*}$, is

$$
P_{s j}^{*}= \begin{cases}\frac{1}{\lambda_{1}^{*}+\lambda_{2}^{*} g_{0 j}}-\frac{K N_{0} B_{c}}{g_{1 j}} & \text { if } \frac{g_{1 j}}{\lambda_{1}^{*}+\lambda_{2}^{*} g_{0 j}} \geq K N_{0} B_{c} \\ 0 & \text { otherwise }\end{cases}
$$

where $\lambda_{1}^{*}$ and $\lambda_{2}^{*}$ are obtained from the following set of equations:

$$
\begin{gathered}
\sum_{j=1}^{M} \int_{\substack{g_{1 j} \\
\lambda_{1}^{*}+\lambda_{2}^{*} g_{0 j}}} \geq K N_{0} B \\
f_{1 j}\left(g_{1 j}\right) f_{0 j}\left(g_{0 j}\right) d g_{0 j} d g_{1 j}=\bar{P}_{s}, \\
\sum_{j=1}^{M} \int_{\frac{g_{1 j}}{\lambda_{1}^{*}+\lambda_{2}^{*} g_{0 j}} \geq K N_{0} B}\left(\frac{K N_{0} B_{c}}{g_{1 j}}\right) \times \\
f_{1 j}\left(g_{1 j}\right) f_{0 j}\left(g_{0 j}\right) d g_{0 j} d g_{1 j}=G Q .
\end{gathered}
$$

Using (7) and (2), $C_{s \mid M}$ is obtained as

$$
\begin{array}{rl}
C_{s \mid M}=\sum_{j=1}^{M} B_{c} \int & \log \left(\frac{g_{1 j}}{\lambda_{1}^{*}+\lambda_{2}^{*} g_{0 j}} \frac{1}{K N_{0} B_{c}}\right) \times \\
\lambda_{1 j}^{*} \lambda_{2}^{*} g_{0 j} & K N_{0} B \\
& \left.g_{1 j}\right) f_{0 j}\left(g_{0 j}\right) d g_{0 j} d g_{1 j} .
\end{array}
$$

For Rayleigh fading, we show that $C_{s \mid M}$ in (10) is (see Appendix A)

$$
C_{s \mid M}=\sum_{j=1}^{M} B_{c} \int_{0}^{\infty} \log \left(\frac{y_{j}}{K N_{0} B_{c}}\right) f_{Y}\left(y_{j}\right) d y_{j},
$$

where

$$
y_{j}=\frac{g_{1 j}}{\lambda_{1}^{*}+\lambda_{2}^{*} g_{0 j}}, j=1,2, \ldots, M,
$$

is a random variable with the probability density function (pdf)

$$
f_{Y}\left(y_{j}\right)=e^{-\lambda_{1}^{*} y_{j}}\left(\frac{\lambda_{1}^{*}}{1+\lambda_{2}^{*} y_{j}}+\frac{\lambda_{2}^{*}}{\left(1+\lambda_{2}^{*} y_{j}\right)^{2}}\right) .
$$

\section{Simulations}

The detailed simulation parameters are presented in Table I. The main performance measure is the normalized maximum achievable capacity (i.e., $C_{s} / B_{c}$ ). Mont-Carlo simulation method with sufficient iterations is adopted.

To show the impact of primary network activity factor on the total achievable capacity, we simulate a DS-CDMA/OFDM system. We then obtain the normalized maximum achievable capacity, $C_{s} / B_{c}$, for a primary network with 50 active users versus primary network activity factor, $\nu$, for different values of $M$. For each value of $M$, the secondary service selects the first $M$ channels sorted based on $g_{1 i} / g_{0 i}$ in descending order. Note that, the best sub-channel can be considered as the one which achieves the maximum capacity and imposes the minimum interference at the same time i.e., $\max _{i} g_{1 i} / g_{0 i}$. Fig. 2 shows the normalized maximum achievable capacity (i.e., $C_{s} / B_{c}$ ) for a single service network with 50 active users
TABLE I

SIMULATION PARAMETERS

\begin{tabular}{l|c}
\hline Parameter & Value \\
\hline Shadowing Coherence Time & $150 \mathrm{~ms}$ \\
Fading Coherence Time & $3 \mathrm{~ms}$ \\
Cell radius & $1000 \mathrm{~m}$ \\
Spectrum Bandwidth $(B)$ & $3.84 \mathrm{MHz}$ \\
$N$ & 64 \\
$B_{c}$ & $60 \mathrm{KHz}$ \\
Standard dev. of Log-Normal shadowing & $8 \mathrm{~dB}$ \\
Propagation loss exponent & 4 \\
Background noise density $\left(N_{0}\right)$ & $-174.0 \mathrm{dBm} / \mathrm{Hz}$ \\
$\bar{P}_{s}$ & $1 \mathrm{Watt}$ \\
$G$ & 125 \\
$Q$ & $N_{0} B$ \\
$f$ & 0.5 \\
Minimum required $E_{b} / I_{0}$ & $2 \mathrm{~dB}$ \\
\hline
\end{tabular}

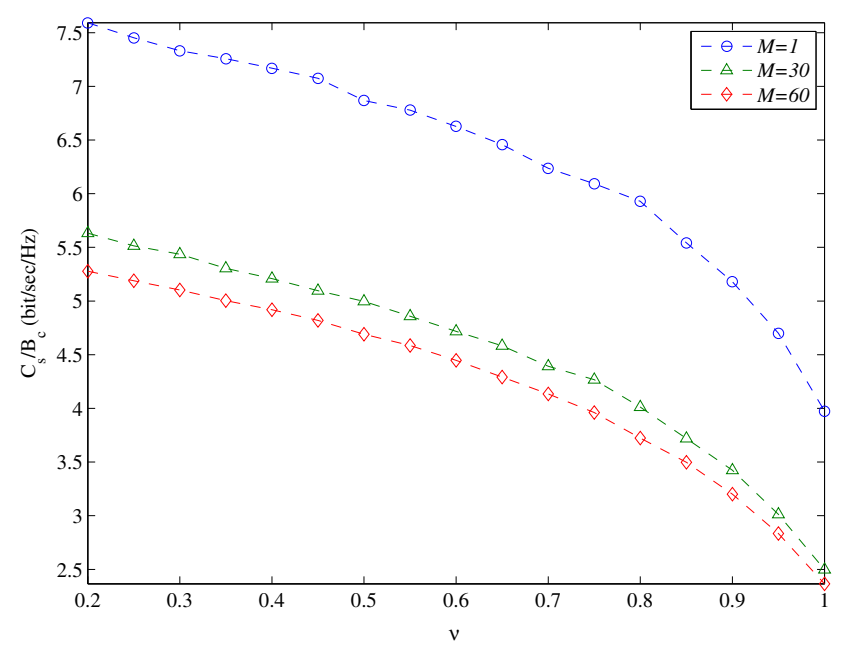

Fig. 2. Normalized maximum achievable capacity, $C_{s} / B_{c}$, versus primary network activity factor, $\nu$, for $M=1, M=30, M=60$.

versus primary network activity factor, $\nu$. In Fig. $2, C_{s} / B_{c}$ is given for $M=1,30,60$.

As it is seen the normalized maximum achievable capacity is significantly increased by decreasing the primary network activity factor. It is worth mentioning that in this simulation the middle-scale temporal variation of the available radio resources is exploited by transmission on the best sub-channels. Note that, since in our simulations we assume perfect CSI availability $C_{s} / B_{c}$ in Fig. 2 is an actual upper-bound for practical cases.

Fig. 3 shows the normalized maximum achievable capacity (i.e., $C_{s} / B_{c}$ ) for a primary network with 50 active users versus primary network activity factor, $\nu$ for different i.i.d. sub-channels $N$. For $M=1$, the secondary service selects a sub-channel with maximum $g_{1 i} / g_{0 i}$. As it is seen by increasing $N$ the normalized maximum achievable capacity is also approximately increased in a linear fashion.

In both results presented above we made an idealistic assumption, in which the primary network allocates a fixed 


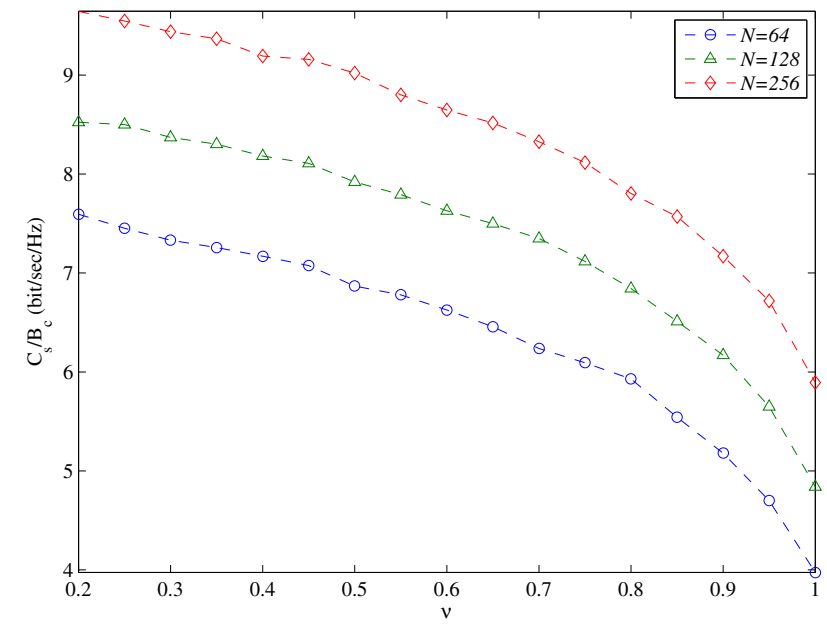

Fig. 3. Normalized maximum achievable capacity, $C_{s} / B_{c}$, versus primary network activity factor, $\nu$, for $M=1$ and $N=64, N=128, N=256$.

portion of its available radio resources to the secondary service by considering a fixed $Q$. However, in practice the value of $Q$ should be estimated based on the activity of the users in the primary network and indeed could be lower if the primary network activity is increased. Taking this fact into consideration, the results presented in Fig. 2 and Fig. 3 acts as an upper-bounds.

\section{Vi. Conclusions and Practical Considerations}

In this paper, we considered a DS-CDMA/OFDMA spectrum sharing system, in which the interference level in the receiver of the primary user is the main constraint for the secondary service transmitter. In this system, the primary network activity reflects itself in the interference level; the more active the primary service, the greater the level of interference is. Formulating this effect in the optimization problem for maximum achievable capacity, we obtained the maximum achievable capacity. Our analytical results showed that the maximum achievable capacity is significantly increased by decreasing the primary network activity factor. This result was also confirmed by simulations. Since we assumed perfect CSI availability the result we obtained for maximum achievable capacity constitutes an upper-bound for practical cases.

If in DS-CDMA/OFDM, the secondary user is able to adaptively select $M$ out of $N$ sub-channels it would add a new degree of freedom for the secondary service. An idea could be making the selection with the objective of maximizing the achievable capacity and at the same time, minimizing the negative impact on the primary network.

Since in practice both CDMA and most of the OFDM cellular systems are single frequency, the operation of the secondary service in the primary band may impose unexpected interference on the receivers in the adjacent cells. To deal with this issue, one may add new constraints to the optimization problems in (2) or consider a conservative value for $Q$.

\section{APPENDIX A}

Here we obtain the pdf of random variable $y_{j}$ in (11). The probability distribution function of random variable $y_{j}$ is

$$
\begin{aligned}
F_{Y}\left(y_{j}\right) & =\operatorname{Pr}\left\{Y \leq y_{j}\right\} \\
& =\operatorname{Pr}\left\{g_{1 j} \leq y_{j}\left(\lambda_{1}^{*}+\lambda_{2}^{*} g_{0 j}\right)\right\} .
\end{aligned}
$$

Since we assume that the channel fading is Rayleigh, both $g_{0 j}$ and $g_{1 j}$ (which are power gains) are i.i.d. unit-mean random variables with exponential distribution; therefore, the probability distribution function of $y_{j}$ is

$$
F_{Y}\left(y_{j}\right)=\int_{0}^{\infty} \int_{0}^{\left(\lambda_{1}^{*}+\lambda_{2}^{*} g_{0 j}\right) y_{j}} e^{-g_{0 j}} e^{-g_{1 j}} d g_{0 j} d g_{1 j} .
$$

The pdf is then obtained by taking the derivative of (14) with respect to $y_{j}$ as

$$
f_{Y}\left(y_{j}\right)=\int_{0}^{\infty}\left(\lambda_{1}^{*}+\lambda_{2}^{*} g_{0 j}\right) e^{-g_{0 j}} e^{-\left(\lambda_{1}^{*}+\lambda_{2}^{*} g_{0 j}\right) y_{j}} d g_{0 j} .
$$

Integrating by part in (15) yields

$$
\begin{aligned}
& \left.f_{Y}\left(y_{j}\right)=-\lambda_{1}^{*} e^{-\lambda_{1}^{*}} \frac{1}{1+\lambda_{2}^{*} y_{j}} e^{-\left(1+\lambda_{2}^{*} y_{j}\right) g_{0 j}}\right]_{0}^{\infty}- \\
& \left.\lambda_{2}^{*} e^{-\lambda_{1}^{*}}\left(\frac{g_{0 j}}{1+\lambda_{2}^{*} y_{j}}-\frac{1}{\left(1+\lambda_{2}^{*} y_{j}\right)^{2}}\right) e^{-\left(1+\lambda_{2}^{*} y_{j}\right) g_{0 j}}\right]_{0}^{\infty} .
\end{aligned}
$$

Thus,

$$
f_{Y}\left(y_{j}\right)=e^{-\lambda_{1}^{*} y_{j}}\left(\frac{\lambda_{1}^{*}}{1+\lambda_{2}^{*} y_{j}}+\frac{\lambda_{2}^{*}}{\left(1+\lambda_{2}^{*} y_{j}\right)^{2}}\right) .
$$

\section{REFERENCES}

[1] "Spectrum policy task force report," Federal Communications Commission, Tech. Rep., November 2002, [online] Available: http://hraunfoss.fcc.gov/edocs_public/attachmatch/DOC-228542A1.pdf.

[2] J. M. Peha, "Approaches to spectrum sharing," IEEE Communications Magazine, pp. 10-12, February 2005.

[3] Q. Zhao and B. Sadler, "A survey of dynamic spectrum access: Signal processing, networking, and regulatory policy," IEEE Signal Processing Magazine, vol. 24, no. 3, pp. 79-89, May 2007.

[4] X. Liu and S. N. Shankar, "Sensing-based opportunistic channel access," ACM Journal on Mobile Networks and Applications (MONET), vol. 11, no. 4, pp. 577-591, August 2006.

[5] M. Gastpar, "On capacity under receive and spatial spectrum-sharing constraints," IEEE Transactions on Information Theory, vol. 53, no. 2, pp. 471-487, February 2007.

[6] S. A. Jafar and S. Srinivasa, "Capacity limits of cognitive radio with distributed and dynamic spectral activity," IEEE Journal on Selected Areas in Communications, vol. 25, no. 3, pp. 529-537, April 2007.

[7] A. Ghasemi and E. S. Sousa, "Fundamental limits of spectrum-sharing in fading environments," IEEE Transactions on Wireless Communications, vol. 6, no. 2, pp. 649-658, February 2007.

[8] D. Tse and P. Viswanath, Fundamentals of Wireless Communication. Cambridge University Press, September 2004

[9] K. Navaie and A. R. Sharafat, "A framework for UMTS air interface analysis," Canadian Journal of Electrical and Computer Engineering, vol. 28, no. 2, pp. 113-129, July/October 2003. 